\title{
Usefulness of Plasma Branched-Chain Amino Acid Analysis in Predicting Outcomes of Patients with Nonischemic Dilated Cardiomyopathy
}

\author{
Yuki Kimura, ${ }^{1}$ MD, Takahiro Okumura, ${ }^{1}$ MD, Shingo Kazama, ${ }^{1}$ MD, Naoki Shibata, ${ }^{1}$ MD, \\ Hideo Oishi, ${ }^{1}$ MD, Yoshihito Arao, ${ }^{1}$ MD, Tasuku Kuwayama, ${ }^{1}$ MD, Hiroo Kato, ${ }^{1}$ MD, \\ Shogo Yamaguchi, ${ }^{1} \mathrm{MD}$, Hiroaki Hiraiwa, ${ }^{1} \mathrm{MD}$, Toru Kondo, ${ }^{1} \mathrm{MD}$, \\ Ryota Morimoto, ${ }^{1} \mathrm{MD}$ and Toyoaki Murohara, ${ }^{1} \mathrm{MD}$
}

\begin{abstract}
Summary
The metabolism of branched-chain amino acids (BCAAs) is reported to change in heart failure (HF) and correlate with cardiac function. However, the effect of BCAAs on HF remains controversial. We investigate the prognostic value of the plasma BCAA level in nonischemic dilated cardiomyopathy (NIDCM).

This study enrolled 39 NIDCM patients, who underwent plasma amino acid (AA) analysis. The ratio of BCAAs to total AAs was calculated. All patients were divided into two groups at the median of BCAA/total AA ratio; high BCAA/total AA group $(\geq 0.15, n=20)$ and low BCAA/total AA group $(<0.15, n=19)$. A cardiac event was defined as a composite of cardiac death, hospitalization for worsening HF, and lethal arrhythmia.

The mean age was $51.1 \pm 12.3$ years and left ventricular ejection fraction (LVEF) was $32.7 \pm 10.1 \%$. In the low BCAA/total AA group, the body mass index and the total cholesterol level were lower than in the high BCAA/total AA group. The BCAA/total AA ratio was positively correlated with $\mathrm{LVEF}(r=0.35, P=0.031)$ and negatively correlated with brain natriuretic peptide $(r=-0.37, P=0.020)$. The low BCAA/total AA group had a lower cardiac event-free rate $(5$-year: $100 \%$ versus $73 \% ; P=0.019)$. In univariate analysis, angiotensin converting enzyme inhibitor or angiotensin II receptor blocker (hazard ratio: $0.045, P=0.0014$ ), hemoglobin (hazard ratio: 0.49 per $1 \mathrm{~g} / \mathrm{dL}, P=0.0022$ ), and BCAA/total AA ratio $<0.15$ (hazard ratio: not available, $P=$ $0.0066)$ were major predictors for cardiac events.
\end{abstract}

The BCAA/total AA ratio might be a useful predictor for future cardiac events in patients with NIDCM.

(Int Heart J 2020; 61: 739-747)

Key words: Metabolism, Heart failure, Nutrition, Prognosis

$\mathrm{T}$ he average heart can use several substrates, such as fatty acids, glucose, ketone bodies, and amino acids (AAs), to meet high energy demands for continuous contraction. These substrates' metabolism changes dramatically in patients with heart failure (HF) ${ }^{1,2)}$ For instance, the primary substrate used for energy switches to glucose from fatty acids in a failing heart. ${ }^{2,3)}$ Additionally, a change in the metabolism of branchedchain amino acids (BCAAs) has been reported in a failing heart. ${ }^{4,5)}$ BCAAs, including leucine, isoleucine, and valine, are essential AAs and metabolized mainly in the skeletal muscles and heart. Almost all other AAs are metabolized in the liver. Within the BCAA catabolic cascade, BCAAs are first converted into branched-chain $\alpha$-keto-acids (BCKAs) by branched-chain amino-transferase. Following that, BCKAs are oxidized by the branched-chain $\alpha$-keto acid dehydrogenase (BCKD) complex and eventually degraded into acetyl-coenzyme A (CoA) or succinyl-CoA. ${ }^{4)}$
In failing hearts, such as in HF or myocardial infarction (MI), the activity of the BCKD complex decreases, and BCAAs and BCKAs accumulate in the tissue. ${ }^{4.5)}$ The increase of BCAAs and BCKAs leads to cardiac remodeling and dysfunction through the mechanistic target of rapamycin (mTOR) activation and reactive oxygen species (ROS) ${ }^{4,5)}$ Indeed, a high plasma BCAA level is a risk factor for cardiac events in patients with ischemic heart disease. ${ }^{(-9)}$

However, Hakuno, et al. reported that Fischer's ratio [BCAA/aromatic AA (AAA) ratio] correlated significantly with cardiac function in $\mathrm{HF}$ with reduced ejection fraction (HFrEF). ${ }^{10)}$ Additionally, several clinical trials have demonstrated the benefit of AA supplementation containing BCAAs in HF patients. ${ }^{11-14)}$ For instance, oral intake of AAs reportedly improve exercise capacities. ${ }^{12-14)}$ Furthermore, BCAA treatment decreases heart rate, preserves cardiac function, and prolongs survival in HFrEF model

From the ${ }^{1}$ Department of Cardiology, Nagoya University Graduate School of Medicine, Nagoya, Japan.

This research was supported by the Suzuken Memorial Foundation, Japan

Address for correspondence: Takahiro Okumura, MD, Department of Cardiology, Nagoya University Graduate School of Medicine, 65 Tsurumai-cho,

Showa-ku, Nagoya, Aichi 466-8550, Japan. E-mail: takaoku@med.nagoya-u.ac.jp

Received for publication January 6, 2020. Revised and accepted April 1, 2020.

Released in advance online on J-STAGE July 18, 2020.

doi: 10.1536/ihj.20-010

All rights reserved by the International Heart Journal Association. 
rats. ${ }^{11}$

Thus, the effect of BCAAs on HF remains controversial. The previous clinical trials consisted of several etiologies of HF, including ischemic heart disease. This variety of etiologies may have resulted in the different observed effects of BCAAs. Therefore, this study's purpose was to investigate the impact of plasma BCAAs on patients with nonischemic dilated cardiomyopathy (NIDCM).

\section{Methods}

Patient population: We included 39 consecutive patients newly diagnosed as NIDCM at our institute between January 2012 and March 2018. NIDCM was defined as previously described. ${ }^{15)}$ In brief, NIDCM was the presence of both a reduced left ventricular ejection fraction $(<50 \%$ as determined by echocardiography or contrast left ventriculography) and a dilated left ventricular cavity, in the absence of coronary artery disease, valvular heart disease, or secondary cardiac muscle disease caused by any known systemic condition, as determined by endomyocardial biopsy. Blood samples for amino acid analysis were collected from all patients. Also, they underwent echocardiography for a cardiac function assessment and right heart catheterization for a hemodynamic assessment.

AA analysis in blood samples: Fasting blood samples in the morning were collected at the timing of the first NIDCM diagnosis and chronic stable phase in HF. The plasma AA analysis was performed by liquid chromatography-mass spectrometry (SRL Inc., Tokyo, Japan). We measured the plasma levels of 39 kinds of AAs (taurine, aspartic acid, hydroxyproline, threonine, serine, asparagine, glutamic acid, glutamine, sarcosine, $\alpha$ aminoadipic acid, proline, glycine, alanine, citrulline, $\alpha$ aminobutyric acid, valine, cystine, cystathionine, methionine, isoleucine, leucine, tyrosine, phenylalanine, $\gamma$ amino $\beta$-hydroxy butyric acid, $\beta$-alanine, $\beta$-amino-isobutyric acid, $\gamma$-aminobutyric acid, monoethanolamine, homocysteine, histidine, 3-methylhistidine, 1-methylhistidine, carnosine, anserine, tryptophan, hydroxylysine, ornithine, lysine, and arginine). Plasma concentration of BCAAs (valine, isoleucine, and leucine) is affected by sex and physical constitution. ${ }^{16)}$ Therefore, we calculated the ratio of BCAAs to total AAs. The normal range of BCAA/total AA ratio was 0.11-0.18 Other laboratory data, such as hemoglobin $(\mathrm{Hb})$, aspartate aminotransferase, alanine aminotransferase, total bilirubin (T-Bil), blood urea nitrogen, creatinine, total protein, albumin (Alb), total cholesterol (TC), sodium, and brain natriuretic peptide (BNP), were measured simultaneously with the AA analysis. Additionally, we calculated the geriatric nutritional risk index (GNRI) to evaluate nutritional status. ${ }^{17)}$ GNRI was calculated using the formula: $[14.89 \times$ Alb $(\mathrm{g} / \mathrm{dL})]+[41.7 \times$ (weight/ideal weight)]. ${ }^{17)}$

Echocardiography: Echocardiographic findings [left ventricular ejection fraction (LVEF), left ventricular diastolic dimension (LVDd), left ventricular systolic dimension, interventricular septal thickness (IVST), posterior wall thickness (PWT), left ventricular mass index (LVMI), left atrial dimension, velocity of mitral annulus early diastolic motion (e'), and E/e' ratio] were measured following the guidelines of the American Society of Echocardiography. ${ }^{18)}$ LVEF was measured using the modified-Simpson method, and the tissue Doppler indices of e' were measured at the septal basal region. LVMI was calculated at end-diastole using the formula of Devereux, et al.: $(0.8 \times$ $\left.\left[1.04 \times\left\{(\operatorname{LVDd}+\text { IVST }+ \text { PWT })^{3}-\operatorname{LVDd}^{3}\right\}\right]+0.6\right) /$ body surface area. ${ }^{19,20)}$

Cardiac catheterization and endomyocardial biopsy: All patients underwent coronary angiography and endocardial biopsy to rule out ischemic heart disease and secondary cardiomyopathy. ${ }^{21)}$ Additionally, hemodynamics were evaluated by right heart catheter and included pulmonary wedge pressure, pulmonary artery pressure, right ventricular pressure, right arterial pressure, and cardiac index which was calculated using the thermodilution method.

Abdominal computed tomography: If patients performed abdominal computed tomography (CT), the skeletal muscle mass was evaluated using the psoas muscle mass index (PMI). ${ }^{22)}$ The cross-sectional area of the bilateral psoas muscles was measured using manual tracing at the umbilical level, and the PMI was calculated using the formula: cross-sectional area of bilateral psoas muscle/ height $t^{2}\left(\mathrm{~cm}^{2} / \mathrm{m}^{2}\right){ }^{22)}$

Treatment after diagnosis of NIDCM: After NIDCM diagnosis, all patients received optimal medical therapy according to standard HF guidelines, ${ }^{23-27)}$ including $\beta$ blocker, angiotensin converting enzyme inhibitor (ACEi) or angiotensin II receptor blocker (ARB), and mineralocorticoid receptor antagonists (MRA). Diuretics were administered for patients with symptoms and/or signs of congestion. An implantable cardioverter defibrillator (ICD) was used as primary prevention in patients with nonsustained ventricular tachycardia and a reduced LVEF of more than $35 \%$. Additionally, if patients had a broadened QRS complex or needed ventricular pacing, cardiac resynchronization therapy (CRT) was considered.

Prognostic analysis: We divided the patients into two groups based on the median BCAA/total AA ratio: high BCAA/total AA group $(\geq 0.15)$ and low BCAA/total AA group $(<0.15)$. The primary endpoint was defined as the composite cardiac events of cardiac death, hospitalization for worsening HF, and lethal arrhythmia (hospitalization for sustained ventricular tachycardia or ventricular fibrillation). The event-free survival rates after diagnosis of NIDCM were compared between the high and low BCAA/total AA groups. Furthermore, the univariate analysis identified prognostic predictors for cardiac events. Ethics: The study protocol was approved by the Ethics Review Board of Nagoya University School of Medicine (approved research number, 2006-0359 and 2017-0031) according to the ethical guidelines of the 1975 Declaration of Helsinki and its amendments. Written informed consent was obtained from all subjects, and the patient records were anonymized before analysis.

Statistical methods: Continuous variables were expressed as means \pm standard deviations or medians and interquartile range. Categorical variables were expressed as numbers and percentages. When comparing the two groups, Pearson's chi-square test or Fisher's exact test were used 
Table I. Clinical Characteristics of Patients

\begin{tabular}{|c|c|c|c|c|}
\hline & $\begin{array}{c}\text { All } \\
(n=39)\end{array}$ & $\begin{array}{l}\text { High BCAA/ } \\
\text { total AA group } \\
\quad(n=20)\end{array}$ & $\begin{array}{l}\text { Low BCAA/ } \\
\text { total AA group } \\
\quad(n=19)\end{array}$ & $P$ value \\
\hline Age, years & $51.1 \pm 12.3$ & $48.3 \pm 11.5$ & $54.0 \pm 12.9$ & 0.15 \\
\hline Male, $n(\%)$ & $29(74)$ & $17(85)$ & $12(63)$ & 0.12 \\
\hline BMI, kg/m² & $24.8 \pm 5.3$ & $27.3 \pm 5.7$ & $22.2 \pm 3.4$ & 0.002 \\
\hline \multicolumn{5}{|l|}{ Comorbidity, $n(\%)$} \\
\hline Hypertension & $10(26)$ & $7(35)$ & $3(16)$ & 0.17 \\
\hline Diabetes mellitus & $6(15)$ & $4(20)$ & $2(11)$ & 0.41 \\
\hline Dyslipidemia & $13(33)$ & $11(55)$ & $2(11)$ & 0.003 \\
\hline Atrial fibrillation & $5(14)$ & $2(10)$ & $3(16)$ & 0.54 \\
\hline Smoking, $n(\%)$ & $15(38)$ & $9(45)$ & $6(32)$ & 0.39 \\
\hline NYHA classification (I/II/III/IV), $n$ & $29 / 8 / 2 / 0$ & $17 / 3 / 0 / 0$ & $12 / 5 / 2 / 0$ & 0.075 \\
\hline \multicolumn{5}{|l|}{ Treatment, $n(\%)$} \\
\hline ACEi/ARB & $28(72)$ & $15(75)$ & $13(68)$ & 0.65 \\
\hline$\beta$-blocker & $26(67)$ & $14(70)$ & $12(63)$ & 0.65 \\
\hline MRA & $24(62)$ & $13(65)$ & $11(58)$ & 0.21 \\
\hline \multicolumn{5}{|l|}{ Device implantation } \\
\hline ICD & $2(5)$ & $0(0)$ & $2(11)$ & 0.14 \\
\hline CRT & $2(5)$ & $0(0)$ & $2(11)$ & 0.14 \\
\hline
\end{tabular}

Data are mean \pm standard deviation or median and interquartile range. BCAA indicates branchedchain amino acid; BMI, body mass index; NYHA, New York Heart Association; ACEi, angiotensin-converting enzyme inhibitor; ARB, angiotensin II receptor blocker; MRA, mineralocorticoid receptor antagonist; ICD, implantable cardioverter defibrillator; and CRT, cardiac resynchronization therapy.

for categorical variables and the unpaired $t$-test. The Wilcoxon rank-sum test was used for continuous variables, as appropriate. The correlations between two parameters were carried out using Pearson's product-moment. Eventfree survival rates were computed by the Kaplan-Meier method and compared using the log-rank test. Univariate analysis for the risk factors associated with composite cardiac events was performed, and the hazard ratios (HRs) and $95 \%$ confidence intervals (CIs) were computed using the Cox-proportional hazard model. Receiver-operating characteristic (ROC) curve analysis was used to assess the clinical utility of the BCAA/total AA ratio for predicting composite cardiac events. The best cutoff value was defined as the point with the highest sum of sensitivity and specificity. A $P<0.05$ was considered statistically significant in all analyses. All statistical analyses were performed using JMP Pro version 11.0 (SAS Institute, Cary, NC, USA).

\section{Results}

Patient characteristics and baseline data: Baseline patient characteristics and treatment are summarized in Table I. The mean age was $51.1 \pm 12.3$ years, and $74 \%$ of the patients were male. In the low BCAA/total AA group, body mass index (BMI) and the history of dyslipidemia were lower than in the high BCAA/total AA group. There were no significant differences among the other comorbidities, New York Heart Association functional class, medication, and device implantation. Furthermore, the administration rates of $\beta$-blockers (100\% versus $100 \%)$, ACEis/ARBs (85\% versus $74 \% ; P=0.38)$, and MRAs (75\% versus $79 \% ; P=0.77$ ) increased during the followup period in both the high and low BCAA/total AA groups. However, the usage rates of ICD and CRT in low BCAA/total AA group were increased during follow-up period compared to those in high BCAA/total AA group (ICD: $5 \%$ versus $37 \% ; P=0.014$, and CRT: $5 \%$ versus $32 \% ; P=0.031)$.

Table II shows clinical data at baseline. The mean LVEF was $32.7 \pm 10.1 \%$, and the median BNP level was $118(57,334) \mathrm{pg} / \mathrm{mL}$. The TC level was lower and PWT was thinner in low BCAA/total AA group $(199 \pm 35 \mathrm{mg}$ / $\mathrm{dL}$ versus $171 \pm 31 \mathrm{mg} / \mathrm{dL} ; P=0.012$, and $9.0 \pm 1.4 \mathrm{~mm}$ versus $8.0 \pm 1.7 \mathrm{~mm} ; P=0.017$, respectively). There were no significant differences in other laboratory data and echocardiographic parameters between the two groups. However, GNRI was lower in low BCAA/total AA group $(112.9 \pm 12.4$ versus $100.2 \pm 12.3 ; P=0.0029)$. Additionally, no difference was shown in hemodynamic data in right heart catheterization.

With respect to the AA analysis, the plasma BCAA level was lower in the low BCAA/total AA group than in the high BCAA/total AA group $(500 \mathrm{nmol} / \mathrm{mL}$ versus 381 $\mathrm{nmol} / \mathrm{mL} ; P<0.0001)$, leading to a lower Fischer's ratio and BCAA/total AA ratio (3.35 versus $3.10 ; P=0.02$, and 0.16 versus $0.14 ; P<0.0001$, respectively). The BCAA/total AA ratio was positively correlated with BMI $(r=0.44, P=0.0047)$, TC $(r=0.45, P=0.0041)$, GNRI $(r=0.45, P=0.0045)$ and LVEF $(r=0.35, P=0.031)$. Furthermore, the BCAA/total AA ratio was negatively correlated with the T-Bil level $(r=-0.43, P=0.0076)$ and BNP $(r=-0.37, P=0.020)$ (Figure 1).

Twenty patients performed abdominal CT and PMI was calculated. PMI was lower in low BCAA/total AA group $[9.38 \pm 1.94(n=10)$ versus $6.69 \pm 1.65(n=10)$; $P=0.0037]$ and positively correlated with the BCAA/total AA ratio $(r=0.49, P=0.028)$. 
Table II. Clinical Data at Baseline

\begin{tabular}{|c|c|c|c|c|}
\hline & $\begin{array}{c}\text { All } \\
(n=39)\end{array}$ & $\begin{array}{l}\text { High BCAA/ } \\
\text { total AA group } \\
\quad(n=20)\end{array}$ & $\begin{array}{l}\text { Low BCAA/ } \\
\text { total AA group } \\
\quad(n=19)\end{array}$ & $P$ value \\
\hline \multicolumn{5}{|l|}{ Laboratory data } \\
\hline $\mathrm{Hb}, \mathrm{g} / \mathrm{dL}$ & $14.0 \pm 1.8$ & $14.5 \pm 1.6$ & $13.5 \pm 1.9$ & 0.067 \\
\hline AST, U/L & $23(18-33)$ & $21(16.5-33)$ & $25(18-37)$ & 0.62 \\
\hline ALT, U/L & $20(16-24)$ & $21(16.8-34.5)$ & $19(14-22)$ & 0.46 \\
\hline T-Bil, mg/dL & $0.8(0.5-1.25)$ & $0.75(0.5-1.08)$ & $0.85(0.68-1.53)$ & 0.17 \\
\hline BUN, mg/dL & $17.5 \pm 4.6$ & $16.8 \pm 3.7$ & $18.4 \pm 5.4$ & 0.27 \\
\hline $\mathrm{Cr}, \mathrm{mg} / \mathrm{dL}$ & $0.90 \pm 0.26$ & $0.90 \pm 0.16$ & $0.91 \pm 0.33$ & 0.91 \\
\hline $\mathrm{TP}, \mathrm{g} / \mathrm{dL}$ & $6.8 \pm 0.6$ & $6.8 \pm 0.5$ & $6.8 \pm 0.7$ & 0.87 \\
\hline $\mathrm{Alb}, \mathrm{g} / \mathrm{dL}$ & $4.1 \pm 0.5$ & $4.2 \pm 0.3$ & $3.9 \pm 0.6$ & 0.16 \\
\hline $\mathrm{TC}, \mathrm{mg} / \mathrm{dL}$ & $186 \pm 35$ & $199 \pm 35$ & $171 \pm 31$ & 0.012 \\
\hline $\mathrm{Na}, \mathrm{mEq} / \mathrm{L}$ & $139(138-141)$ & $140(138-141)$ & $139(138-141)$ & 0.59 \\
\hline $\mathrm{BNP}, \mathrm{pg} / \mathrm{mL}$ & $118(57-334)$ & $90(27-206)$ & $224(108-629)$ & 0.071 \\
\hline GNRI & $106.7 \pm 13.8$ & $112.9 \pm 12.4$ & $100.2 \pm 12.3$ & 0.0029 \\
\hline \multicolumn{5}{|l|}{ Amino acid analysis } \\
\hline BCAAs, $\mathrm{nmol} / \mathrm{mL}$ & $432(381-507)$ & $500(449-523)$ & $381(332-430)$ & $<0.0001$ \\
\hline AAAs, nmol/mL & $137(123-156)$ & $140(127-163)$ & $127(118-147)$ & 0.23 \\
\hline total AAs, $\mathrm{nmol} / \mathrm{mL}$ & $2919(2628-3113)$ & 2964 (2706-3206) & $2892(2583-3144)$ & 0.078 \\
\hline Fischer ratio* & $3.16(3.05-3.59)$ & $3.35(3.12-3.66)$ & $3.10(2.68-3.50)$ & 0.02 \\
\hline $\mathrm{BCAA} /$ total AA ratio & $0.15(0.14-0.16)$ & $0.16(0.16-0.17)$ & $0.14(0.12-0.15)$ & $<0.0001$ \\
\hline \multicolumn{5}{|c|}{ Echocardiographic findings } \\
\hline LVEF, \% & $32.7 \pm 10.1$ & $35.7 \pm 8.0$ & $29.5 \pm 11.2$ & 0.052 \\
\hline LVDd, mm & $64.5 \pm 9.4$ & $64.0 \pm 9.7$ & $65.0 \pm 9.4$ & 0.75 \\
\hline LVDs, mm & $54.7 \pm 10.2$ & $53.2 \pm 10.4$ & $56.3 \pm 10.1$ & 0.35 \\
\hline IVST, mm & $8.6 \pm 1.5$ & $9.0 \pm 1.7$ & $8.1 \pm 1.2$ & 0.67 \\
\hline PWT, mm & $8.7 \pm 1.7$ & $9.3 \pm 1.4$ & $8.0 \pm 1.7$ & 0.017 \\
\hline LVMI, $\mathrm{g} / \mathrm{m}^{2}$ & $159 \pm 44$ & $155 \pm 41$ & $164 \pm 45$ & 0.52 \\
\hline $\mathrm{LAD}, \mathrm{mm}$ & $41.3 \pm 7.9$ & $41.6 \pm 7.6$ & $41.1 \pm 8.5$ & 0.85 \\
\hline $\mathrm{e}^{\prime}, \mathrm{cm} / \mathrm{second}$ & $4.7(3.5-6.9)$ & $4.9(3.8-7.0)$ & $4.2(3.3-6.8)$ & 0.56 \\
\hline $\mathrm{E} / \mathrm{e}^{\prime}$ & $13.5(10.6-18.3)$ & $11.2(9.3-15.2)$ & $17.2(12.1-19.8)$ & 0.16 \\
\hline \multicolumn{5}{|l|}{ Hemodynamic parameters } \\
\hline $\mathrm{sBP}, \mathrm{mmHg}$ & $117.9 \pm 25.4$ & $122.5 \pm 27.3$ & $113.2 \pm 23.0$ & 0.26 \\
\hline $\mathrm{dBP}, \mathrm{mmHg}$ & $71.6 \pm 12.4$ & $74.6 \pm 11.6$ & $68.4 \pm 12.8$ & 0.13 \\
\hline HR, beat/minute & $76.6 \pm 12.4$ & $76.3 \pm 10.6$ & $76.9 \pm 14.5$ & 0.87 \\
\hline PCWP, mmHg & $14.0 \pm 7.8$ & $13.0 \pm 7.2$ & $15.2 \pm 8.5$ & 0.39 \\
\hline sPAP, mmHg & $30.3 \pm 10.7$ & $28.8 \pm 10.4$ & $31.9 \pm 11.2$ & 0.37 \\
\hline $\mathrm{dPAP}, \mathrm{mmHg}$ & $12.8 \pm 6.9$ & $12.0 \pm 6.2$ & $13.6 \pm 7.6$ & 0.45 \\
\hline $\mathrm{RVP}, \mathrm{mmHg}$ & $29.8 \pm 10.2$ & $28.9 \pm 10.8$ & $30.7 \pm 9.7$ & 0.58 \\
\hline RAP, mmHg & $5.7 \pm 3.6$ & $5.5 \pm 3.3$ & $6.1 \pm 4.0$ & 0.61 \\
\hline $\mathrm{CI}, \mathrm{L} /$ minute $/ \mathrm{m}^{2}$ & $2.45 \pm 0.61$ & $2.48 \pm 0.62$ & $2.42 \pm 0.62$ & 0.76 \\
\hline
\end{tabular}

Data are mean \pm standard deviation or median and interquartile range. BCAA indicates branched-chain amino acid; Hb, hemoglobin; AST, aspartate aminotransferase; ALT, alanine aminotransferase; T-Bil, total bilirubin; BUN, blood urea nitrogen; Cr, creatinine; TP, total protein; Alb, albumin; TC, total cholesterol; Na, sodium concentration; BNP, brain natriuretic peptide; GNRI, geriatric nutritional risk index; AAA, aromatic amino acid; LVEF, left ventricular ejection fraction; LVDd, left ventricular diastolic dimension; LVDs, left ventricular systolic dimension; IVST, interventricular septal thickness; PWT, posterior wall thickness; LVMI, left ventricular mass index; LAD, left atrial dimension; e', velocity of mitral annulus early diastolic motion; E, mitral peak E-wave velocity; $\mathrm{sBP}$, systolic blood pressure; $\mathrm{dBP}$, diastolic blood pressure; HR, heart rate; PCWP, pulmonary wedge pressure; SPAP, systolic pulmonary artery pressure; dPAP, diastolic pulmonary artery pressure; RVP, right ventricular pressure; RAP, right arterial pressure; and CI, cardiac index. *Fischer ratio is the ratio of BCAAs to AAAs.

Follow-up of cardiac events: All patients were followedup for a mean of $1297 \pm 691$ days $(1166 \pm 632$ days versus $1434 \pm 740$ days in the high and low BCAA/total AA groups, respectively; $P=0.23)$. During the follow-up period, six cardiac events ( 0 cardiac death, five hospitalizations for worsening HF, and one lethal arrhythmia) were experienced. Kaplan-Meier survival analysis revealed that the event-free survival rate was significantly lower in the low BCAA/total AA group than in the high BCAA/total AA group (1-year: $79 \%$ versus $100 \%$, 5-year: $73 \%$ versus $100 \% ; P=0.019$ ) (Figure 2A). On the other hand, the rate of all-cause deaths was not significantly different between the low and high BCAA/total AA groups (1-year: $95 \%$ versus $100 \%$, 5-year: $83 \%$ versus $100 \% ; P=0.082$ ) (Figure 2B).

In univariate analyses, the factors associated with an 
A

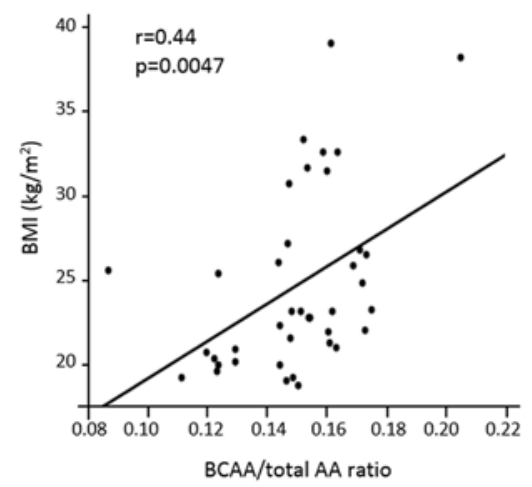

D

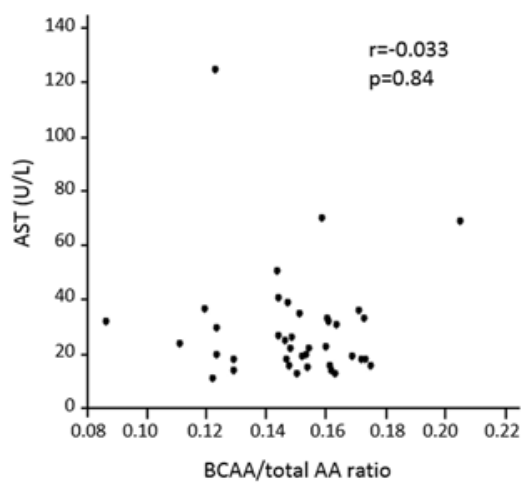

G

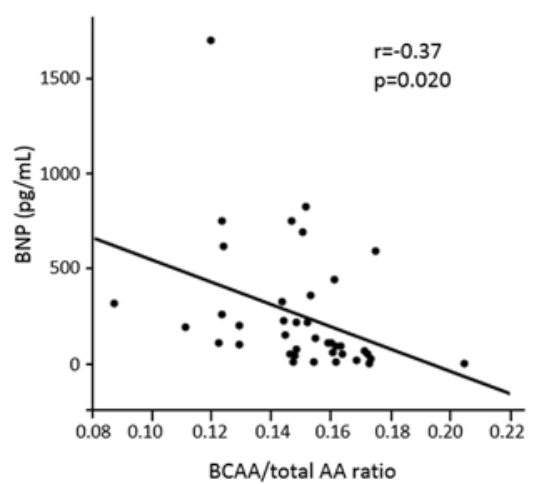

B

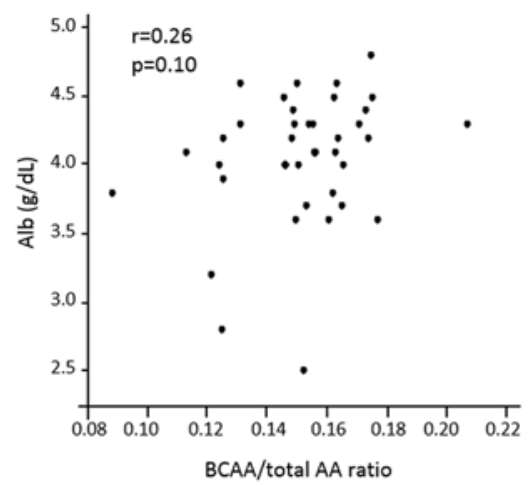

E

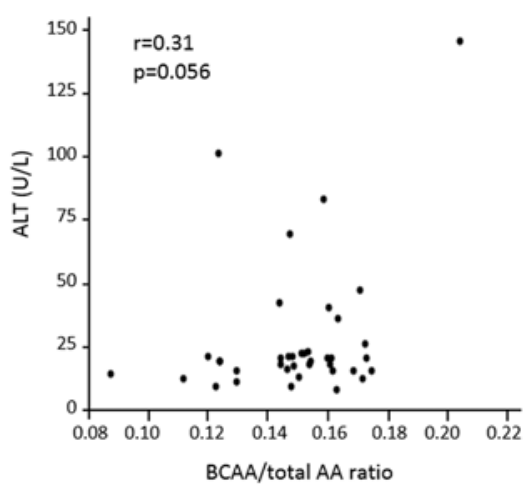

H

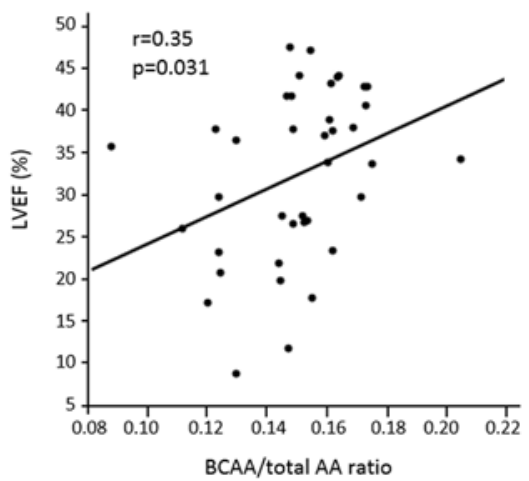

C

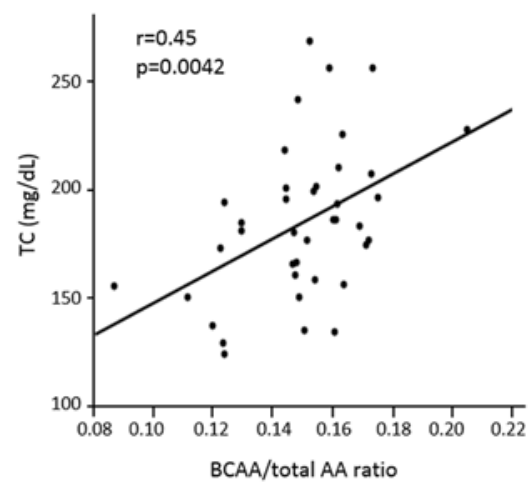

F

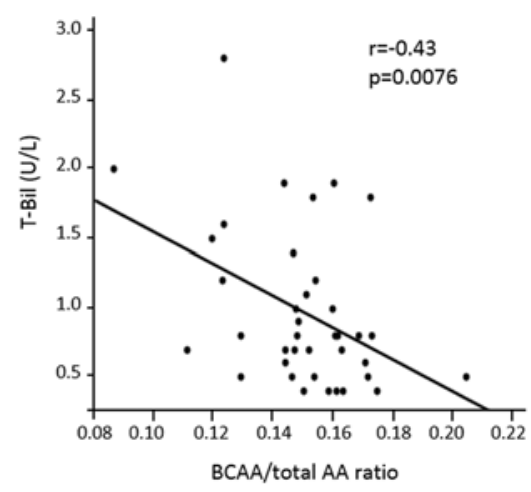

I

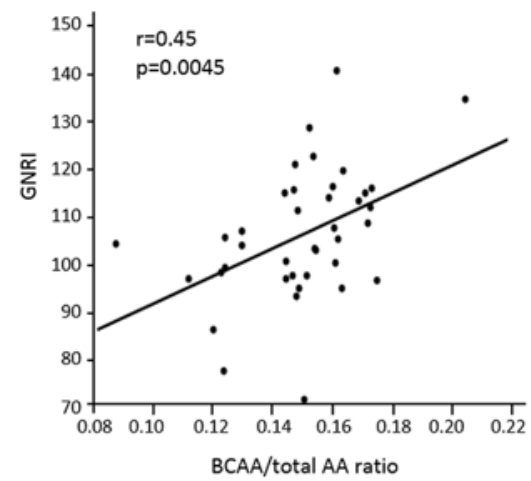

Figure 1. Correlations with BCAA/total AA ratio. The correlations between BCAA/total AA ratio and body mass index (A), albumin (B), total cholesterol $(\mathbf{C})$, aspartate aminotransferase $(\mathbf{D})$, alanine aminotransferase $(\mathbf{E})$, total bilirubin $(\mathbf{F})$, brain natriuretic peptide $(\mathbf{G})$, left ventricular ejection fraction $(\mathbf{H})$, and geriatric nutritional risk index (I). BCAA indicates branched-chain amino acid; AA, amino acid; BMI, body mass index; Alb, albumin; TC, total cholesterol; AST, aspartate aminotransferase; ALT, alanine aminotransferase; T-Bil, total bilirubin; BNP, brain natriuretic peptide; LVEF, left ventricular ejection fraction; and GNRI, geriatric nutritional risk index.

increased risk of composite event were age (HR: 1.09 per 1 year, 95\% CI: $1.01-1.19, P=0.027)$, administration of ACEi/ARB (HR: $0.045,95 \%$ CI: $0.002-0.31, P=0.0014$ ), usage of ICD (HR: $6.22,95 \%$ CI: $1.03-47.28, P=0.047$ ), $\mathrm{Hb}$ level (HR: 0.49 per $\mathrm{Hb} 1 \mathrm{~g} / \mathrm{dL}, 95 \%$ CI: 0.29-0.77, $P$ $=0.0022)$, and BCAA/total AA ratio < 0.15 (HR: not available, $P=0.0066$ ) (Table III). By analyzing the ROC curve, the area under the ROC curve of the BCAA/total AA ratio was 0.75 with the best cutoff point of 0.1497 .

\section{Discussion}

The main findings of this study are: 1) The BCAA/ total AA ratio was positively correlated with BMI, TC, and LVEF, and negatively correlated with T-Bil and BNP; 2) NIDCM patients with low BCAA/total AA ratio had a poor prognosis compared to that with high $\mathrm{BCAA} /$ total AA ratio; 3) Low BCAA/total AA ratio was a predictor of composite cardiac event in patients with NIDCM.

In stressed hearts, the activity of the BCKD complex 
A

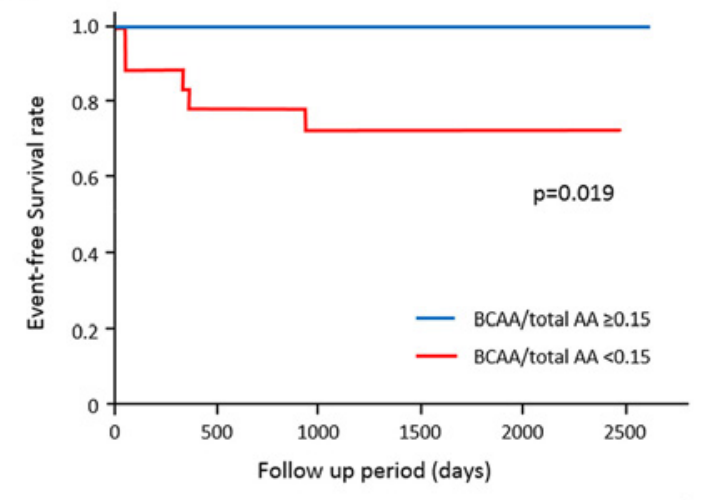

\begin{tabular}{lccc}
\hline & \multicolumn{3}{c}{ Event-free rate $(\mathrm{n})$} \\
\cline { 2 - 4 } & 1 year & 3 year & 5 year \\
\hline BCAA/total AA $\geq 0.15$ & $1.00(20)$ & $1.00(12)$ & $1.00(3)$ \\
BCAA/total AA $<0.15$ & $0.79(15)$ & $0.73(14)$ & $0.73(9)$ \\
\hline
\end{tabular}

B

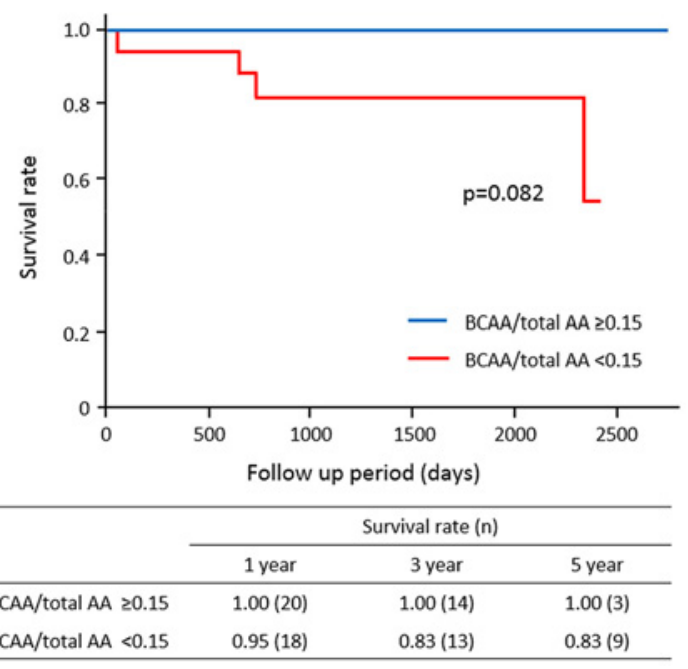

Figure 2. Kaplan-Meier curves for event-free rate in the high and low BCAA/total AA groups. A: Composite cardiac event. B: All-cause death. BCAA indicates branched-chain amino acid; AA, amino acid. A composite cardiac event was defined as cardiac death, hospitalization for worsening HF, and lethal arrhythmia. Lethal arrhythmia was defined as hospitalization for sustained ventricular tachycardia or ventricular fibrillation

Table III. Univariate Regression Analysis for a Composite Cardiac Event

\begin{tabular}{|c|c|c|}
\hline Factors & $\mathrm{HR}(95 \% \mathrm{CI})$ & $P$ value \\
\hline Age (per 1 year) & $1.09(1.01-1.19)$ & 0.027 \\
\hline BMI $\left(\right.$ per $\left.1 \mathrm{~kg} / \mathrm{m}^{2}\right)$ & $0.91(0.68-1.10)$ & 0.39 \\
\hline Hypertension & $1.96(0.26-11.83)$ & 0.47 \\
\hline Diabetes mellitus & $4.59(0.60-28.12)$ & 0.13 \\
\hline Atrial fibrillation & $1.65(0.08-11.56)$ & 0.67 \\
\hline Smoking & $1.10(0.14-6.65)$ & 0.92 \\
\hline NYHA classification $\geq$ II & $4.98(0.82-37.94)$ & 0.079 \\
\hline ACEi/ARB & $0.045(0.002-0.31)$ & 0.0014 \\
\hline$\beta$ blocker & $\mathrm{n} / \mathrm{a}$ & $\mathrm{n} / \mathrm{a}$ \\
\hline MRA & $\mathrm{n} / \mathrm{a}$ & 0.091 \\
\hline ICD & $6.22(1.03-47.28)$ & 0.047 \\
\hline CRT & $3.06(0.40-18.50)$ & 0.25 \\
\hline $\mathrm{Hb}($ per $1 \mathrm{~g} / \mathrm{dL})$ & $0.49(0.29-0.77)$ & 0.0022 \\
\hline $\operatorname{AST}($ per $1 \mathrm{U} / \mathrm{L})$ & $1.00(0.95-1.03)$ & 0.85 \\
\hline T-Bil (per 1 mg/dL) & $1.77(0.39-5.73)$ & 0.41 \\
\hline $\mathrm{Cr}($ per $1 \mathrm{mg} / \mathrm{dL})$ & $15.80(0.78-228.88)$ & 0.069 \\
\hline $\mathrm{Alb}($ per $1 \mathrm{~g} / \mathrm{dL})$ & $0.21(0.047-1.09)$ & 0.062 \\
\hline TC (per $1 \mathrm{mg} / \mathrm{dL}$ ) & $0.99(0.96-1.02)$ & 0.45 \\
\hline $\mathrm{BNP}($ per $10 \mathrm{pg} / \mathrm{mL})$ & $1.00(0.97-1.02)$ & 0.71 \\
\hline BCAA/total AA ratio $<0.15$ & $\mathrm{n} / \mathrm{a}$ & 0.0066 \\
\hline Fischer's ratio $<3.0$ & $2.3(0.38-13.79)$ & 0.36 \\
\hline LVEF (per 1\%) & $1.02(0.94-1.14)$ & 0.62 \\
\hline $\mathrm{CI}\left(\right.$ per $1 \mathrm{~L} /$ minute $\left./ \mathrm{m}^{2}\right)$ & $1.84(0.43-7.96)$ & 0.40 \\
\hline
\end{tabular}

HR indicates hazard ratio; 95\% CI, 95\% confidence intervals; BMI, body mass index; NYHA, New York Heart Association; $\mathrm{ACEi}$, angiotensin-converting enzyme inhibitor; $\mathrm{ARB}$, angiotensin II receptor blocker; MRA, mineralocorticoid receptor antagonist; ICD, implantable cardioverter defibrillator; CRT, cardiac resynchronization therapy; $\mathrm{Hb}$, hemoglobin; AST, aspartate aminotransferase; T-Bil, total bilirubin; $\mathrm{Cr}$, creatinine; Alb, albumin; TC, total cholesterol; BNP, brain natriuretic peptide; BCAA, branched-chain amino acid; LVEF, left ventricular ejection fraction; $\mathrm{CI}$, cardiac index; and n/a, not available. decreases because of reduced expression of protein phosphatase $2 \mathrm{Cm}$, which activates BCKD. ${ }^{4,5}$ Additionally, BCKD subunit such as E1alfa, E1beta, and E2 are downregulated. ${ }^{5,28}$ Subsequently, BCAAs and BCKAs are elevated within the myocardium in HF patients. ${ }^{29)}$ Accumulation of BCAAs and BCKAs contributes to the pathogenesis of HF by mTOR activation, ROS production, and mitochondrial dysfunction. ${ }^{2,45}$ However, the elevated BCAAs in myocardium does not always lead to an increase in plasma BCAA level. Additionally, the balance of the BCAA level changes dramatically. For instance, the BCAA level in myocardium increases after MI, while the plasma BCAA level decreases during the acute phase of MI and after that gradually increases to baseline. ${ }^{30,31)}$ This change in plasma BCAA level is thought to be affected by enhanced metabolism of BCAAs in skeletal muscle. ${ }^{30)} \mathrm{Ad}-$ ditionally, the difference in the etiology of cardiac diseases may change the meaning of serum BCAAs. In the current study, the BCAA/total AA ratio was correlated with BMI and TC. The elevated levels of these factors are significant risks of developing coronary artery disease and increase the recurrence of a coronary event and death. ${ }^{32-36}$ The etiology was coronary artery disease or ischemic cardiomyopathy in previous articles which reported elevated BCAAs was a risk factor for cardiac event. ${ }^{6-9)}$ For these reasons, the meaning of high BCAAs was different in the current study, including patients with NIDCM.

Because BCAAs are essential AAs which cannot be synthesized in the body, the BCAA level is affected by dietary intake and absorption from the intestine. Indeed, Ottestad, et al. reported that the BCAA level decreased in sarcopenic adults, whereas the non-essential amino acid levels did not change. ${ }^{37)}$ In chronic HF, intestinal function declines because of restricted intestinal perfusion and consequent mucosal edema, enhanced intestinal permeability, and a lack of immunological defense with augmented bac- 
terial biofilm. ${ }^{38,39)}$ Concomitantly, the absorption of proteins declines, leading to a decrease in the BCAA level. ${ }^{40)}$ In the current study, the BCAA/total AA ratio was correlated with BMI, TC, and GNRI. The weight loss and low level of TC are risk factors for mortality in patients with $\mathrm{HF}^{41-43)}$ Additionally, GNRI is a nutrition-related risk index that can classify patients according to a risk of morbidity and mortality associated with malnutrition. ${ }^{17)}$ Therefore, a low BCAA/total AA ratio may reflect poor nutritional condition and indicate a poor prognosis in NIDCM patients.

Furthermore, liver function is important when considering the metabolism of AAs and the plasma BCAA level. In patients with liver dysfunction, the metabolism of AAAs in the liver decreases; on the other hand, the metabolism of BCAAs in skeletal muscles increases. An increase in the plasma AAA level and a decrease in the BCAA level, leads to a low Fischer's ratio. ${ }^{44}$ The Fischer's ratio was reported to decrease in patients with chronic $\mathrm{HF}^{30)}$ and was significantly correlated with cardiac function and the level of BNP. ${ }^{10)}$ In the current study, there was no significant difference in the plasma level of AAAs between the high and low BCAA/total AA groups. This may be because the levels of transaminase were within the normal range and liver dysfunction was not severe in most patients. However, BCAA/total AA ratio was significantly correlated with the level of T-Bil. Therefore, the BCAA/total AA ratio may decrease in patients with mild liver dysfunction. An elevated T-Bil level was associated with HF re-hospitalization, cardiac events, and death in patients with chronic $\mathrm{HF}^{45,46)}$ Based on these reasons, the BCAA/total AA ratio might be able to predict cardiac events in patients with NIDCM in association with intestinal function, nutritional status, and liver function. Indeed $\mathrm{BCAA} /$ total AA ratio was correlated with reliable predictors of prognosis in patients with NIDCM such as BNP and LVEF.

Fischer's ratio was reported to be a useful prognostic marker in HF patients. ${ }^{10,47)}$ However, we selected the $\mathrm{BCAA} /$ total AA ratio to investigate the impact of plasma BCAAs in patients with NIDCM, because Fischer's ratio is affected by the concentration of AAAs. In the current study, the BCAA/total AA ratio was more useful to predict composite cardiac events compared to Fischer's ratio. AAAs are mainly metabolized in a liver; therefore, the level of AAAs is affected by the severity of heart failure rather than the etiology of heart failure. Our patients were in the early stage of heart failure. In our subjects, the metabolism of BCAAs started to change, whereas the liver function still preserved and the plasma level of AAAs did not increase. This might be a reason Fischer's ratio was not a predictor for cardiac events in the current study.

This study has several fundamental limitations. First, this was an observational study and the sample size and number of events were relatively small. Therefore, this study might be prone to statistical errors. Second, the study arm was the only group of patients with NIDCM. We could not compare the BCAA/total AA ratio between healthy controls and NIDCM patients. Third, we did not collect information about the content and amount of meals. We could not evaluate the dietary intake of
BCAAs. Fourth, we could not perform multivariate analysis because of the small number of events. Therefore, this study could not eliminate the cofounding bias. In addition, we could not compare the prognostic value between the BCAA/total AA ratio and known predictors including the Fischer's ratio. A prospective multicenter study with a greater number of cases is needed to determine the usefulness of BCAA/total AA ratio in NIDCM patients. Finally, we could not evaluate the BCAA level in myocardium, therefore the metabolism of BCAAs in myocardium in patients suffering from NIDCM is unclear. We need an additional study to elucidate BCAA metabolism in myocardium in patients with NIDCM.

In conclusion, the evaluation of $\mathrm{BCAA} /$ total $\mathrm{AA}$ ratio by AA analysis might be useful for future cardiac events in patients with HF caused by NIDCM.

\section{Acknowledgments}

Author statement: All authors contributed to the conception, design, critical revision and final approval of this manuscript. YK, SK, NS, HO, YA, TK, HK, SY, HH, and TK acquired data. YK, RM, and TO performed statistical analyses, interpreted the data, and drafted the manuscript under supervision of senior authors TM.

\section{Disclosure}

Conflicts of interest: TO has received research grants from Ono Pharmaceutical Co., Ltd., Bayer Pharmaceutical Co., Ltd., Daiichi-Sankyo Pharma Inc., and Amgen Astellas BioPharma K. K. outside the submitted work. TO received honorariums from Ono Pharmaceutical Co., Ltd., Otsuka Pharmaceutical Co., Ltd., and Medtronic Japan Co., Ltd. TM received lecture fees from Bayer Pharmaceutical Co., Ltd., Daiichi-Sankyo Co., Ltd., Dainippon Sumitomo Pharma Co., Ltd., Kowa Co., Ltd., MSD K. K., Mitsubishi Tanabe Pharma Co., Nippon Boehringer Ingelheim Co., Ltd., Novartis Pharma K. K., Pfizer Japan Inc., Sanofi-aventis K. K., and Takeda Pharmaceutical Co., Ltd. TM received unrestricted research grant for Department of Cardiology, Nagoya University Graduate School of Medicine from Astellas Pharma Inc., Daiichi-Sankyo Co., Ltd., Dainippon Sumitomo Pharma Co., Ltd., Kowa Co., Ltd., MSD K. K., Mitsubishi Tanabe Pharma Co., Nippon Boehringer Ingelheim Co., Ltd., Novartis Pharma K. K., Otsuka Pharma Ltd., Pfizer Japan Inc., Sanofi-aventis K. K., Takeda Pharmaceutical Co., Ltd., and Teijin Pharma Ltd. The other authors declare that they have no conflicts of interest.

\section{References}

1. Ikegami R, Shimizu I, Yoshida Y, Minamino T. Metabolomic analysis in heart failure. Circ J 2017; 82: 10-6.

2. Wende AR, Brahma MK, McGinnis GR, Young ME. Metabolic origins of heart failure. JACC Basic Transl Sci 2017; 2: 297310 .

3. Fukushima A, Milner K, Gupta A, Lopaschuk GD. Myocardial energy substrate metabolism in heart failure: from pathways to therapeutic targets. Curr Pharm Des 2015; 21: 3654-64. 
4. Huang Y, Zhou M, Sun H, Wang Y. Branched-chain amino acid metabolism in heart disease: an epiphenomenon or a real culprit? Cardiovasc Res 2011; 90: 220-3.

5. Sun H, Wang Y. Branched chain amino acid metabolic reprogramming in heart failure. Biochim Biophys Acta 2016; 1862 2270-5.

6. Ruiz-Canela M, Toledo E, Clish CB, et al. Plasma branchedchain amino acids and incident cardiovascular disease in the PREDIMED Trial. Clin Chem 2016; 62: 582-92.

7. Du X, You H, Li Y, et al. Relationships between circulating branched chain amino acid concentrations and risk of adverse cardiovascular events in patients with STEMI treated with PCI. Sci Rep 2018; 8: 15809.

8. Du X, Li Y, Wang Y, et al. Increased branched-chain amino acid levels are associated with long-term adverse cardiovascular events in patients with STEMI and acute heart failure. Life Sci 2018; 209: 167-72.

9. Shah SH, Bain JR, Muehlbauer MJ, et al. Association of a peripheral blood metabolic profile with coronary artery disease and risk of subsequent cardiovascular events. Circ Cardiovasc Genet 2010; 3: 207-14.

10. Hakuno D, Hamba Y, Toya T, Adachi T. Plasma amino acid profiling identifies specific amino acid associations with cardiovascular function in patients with systolic heart failure. PLOS ONE 2015; 10: e0117325.

11. Tanada Y, Shioi T, Kato T, Kawamoto A, Okuda J, Kimura T. Branched-chain amino acids ameliorate heart failure with cardiac cachexia in rats. Life Sci 2015; 137: 20-7.

12. Aquilani R, Opasich C, Gualco A, et al. Adequate energyprotein intake is not enough to improve nutritional and metabolic status in muscle-depleted patients with chronic heart failure. Eur J Heart Fail 2008; 10: 1127-35.

13. Aquilani R, Viglio S, Iadarola P, et al. Oral amino acid supplements improve exercise capacities in elderly patients with chronic heart failure. Am J Cardiol 2008; 101: 104E-10E.

14. Scognamiglio R, Testa A, Aquilani R, Dioguardi FS, Pasini E. Impairment in walking capacity and myocardial function in the elderly: is there a role for nonpharmacologic therapy with nutritional amino acid supplements? Am J Cardiol 2008; 101: 78E$81 \mathrm{E}$.

15. Okumura T, Hirashiki A, Yamada S, et al. Association between cardiopulmonary exercise and dobutamine stress testing in ambulatory patients with idiopathic dilated cardiomyopathy: a comparison with peak $\mathrm{VO}_{2}$ and $\mathrm{VE} / \mathrm{VCO}_{2}$ slope. Int $\mathrm{J}$ Cardiol 2013; 162: 234-9.

16. Merz B, Frommherz L, Rist MJ, Kulling SE, Bub A, Watzl B. Dietary pattern and plasma BCAA-variations in healthy men and women-results from the KarMeN study. Nutrients 2018; 10: E623.

17. Bouillanne O, Morineau G, Dupont C, et al. Geriatric Nutritional Risk Index: a new index for evaluating at-risk elderly medical patients. Am J Clin Nutr 2005; 82: 777-83.

18. Cheitlin MD, Armstrong WF, Aurigemma GP, et al. ACC/AHA/ ASE 2003 guideline update for the clinical application of echocardiography--summary article: a report of the American College of Cardiology/American Heart Association Task Force on Practice Guidelines (ACC/AHA/ASE Committee to Update the 1997 Guidelines for the Clinical Application of Echocardiography). J Am Coll Cardiol 2003; 42: 954-70.

19. Devereux RB, Reichek N. Echocardiographic determination of left ventricular mass in man. Anatomic validation of the method. Circulation 1977; 55: 613-8.

20. Devereux RB, Alonso DR, Lutas EM, et al. Echocardiographic assessment of left ventricular hypertrophy: comparison to necropsy findings. Am J Cardiol 1986; 57: 450-8.

21. Elliott P, Andersson B, Arbustini E, et al. Classification of the cardiomyopathies: a position statement from the European Society of Cardiology Working Group on Myocardial and Pericardial Diseases. Eur Heart J 2008; 29: 270-6.

22. Harada K, Suzuki S, Ishii H, et al. Impact of skeletal muscle mass on long-term adverse cardiovascular outcomes in patients with chronic kidney disease. Am J Cardiol 2017; 119: 1275-80.

23. Yancy CW, Jessup M, Bozkurt B, et al. 2013 ACCF/AHA guideline for the management of heart failure: a report of the American College of Cardiology Foundation/American Heart Association Task Force on Practice Guidelines. Circulation 2013; 128: e240-327.

24. Ponikowski P, Voors AA, Anker SD, et al. 2016 ESC Guidelines for the diagnosis and treatment of acute and chronic heart failure: the Task Force for the diagnosis and treatment of acute and chronic heart failure of the European Society of Cardiology (ESC). Developed with the special contribution of the Heart Failure Association (HFA) of the ESC. Eur J Heart Fail 2016; 18: 891-975.

25. Komajda M. Current challenges in the management of heart failure. Circ J 2015; 79: 948-53.

26. Fonarow GC. Improving quality of care and outcomes for heart failure. Circ J 2011; 75: 1783-90.

27. Ohsaka T, Inomata T, Naruke T, et al. Clinical impact of adherence to guidelines on the outcome of chronic heart failure in Japan. Int Heart J 2008; 49: 59-73.

28. Sun H, Olson KC, Gao C, et al. Catabolic defect of branchedchain amino acids promotes heart failure. Circulation 2016; 133: 2038-49.

29. Shimazu T, Hirschey MD, Newman J, et al. Suppression of oxidative stress by $\beta$-hydroxybutyrate, an endogenous histone deacetylase inhibitor. Science 2013; 339: 211-4.

30. Wang W, Zhang F, Xia Y, et al. Defective branched chain amino acid catabolism contributes to cardiac dysfunction and remodeling following myocardial infarction. Am J Physiol Heart Circ Physiol 2016; 311: H1160-9.

31. Tsuji S, Koyama S, Taniguchi R, Fujiwara T, Fujiwara H, Sato Y. Nutritional status of outpatients with chronic stable heart failure based on serum amino acid concentration. J Cardiol 2018; 72: 458-65.

32. Chei CL, Iso H, Yamagishi K, Inoue M, Tsugane S. Body mass index and weight change since 20 years of age and risk of coronary heart disease among Japanese: the Japan Public Health Center-Based Study. Int J Obes (Lond) 2008; 32: 144-51.

33. Cui R, Iso H, Toyoshima H, et al. Body mass index and mortality from cardiovascular disease among Japanese men and women: the JACC study. Stroke 2005; 36: 1377-82.

34. Okamura T, Tanaka H, Miyamatsu N, et al. The relationship between serum total cholesterol and all-cause or cause-specific mortality in a 17.3-year study of a Japanese cohort. Atherosclerosis 2007; 190: 216-23.

35. Matsuzaki M, Kita T, Mabuchi H, et al. Large scale cohort study of the relationship between serum cholesterol concentration and coronary events with low-dose simvastatin therapy in Japanese patients with hypercholesterolemia. Circ J 2002; 66: 1087-95.

36. Honjo K, Iso H, Tsugane S, et al. The effects of smoking and smoking cessation on mortality from cardiovascular disease among Japanese: pooled analysis of three large-scale cohort studies in Japan. Tob Control 2010; 19: 50-7.

37. Ottestad I, Ulven SM, Øyri LKL, et al. Reduced plasma concentration of branched-chain amino acids in sarcopenic older subjects: a cross-sectional study. Br J Nutr 2018; 120: 445-53.

38. Sandek A, Bauditz J, Swidsinski A, et al. Altered intestinal function in patients with chronic heart failure. J Am Coll Cardiol 2007; 50: 1561-9.

39. Sandek A, Bjarnason I, Volk HD, et al. Studies on bacterial endotoxin and intestinal absorption function in patients with chronic heart failure. Int J Cardiol 2012; 157: 80-5.

40. Sandek A, Anker SD, von Haehling S. The gut and intestinal bacteria in chronic heart failure. Curr Drug Metab 2009; 10: 228.

41. Horwich TB, Fonarow GC, Hamilton MA, MacLellan WR, Woo MA, Tillisch JH. The relationship between obesity and mortality in patients with heart failure. J Am Coll Cardiol 2001; 38: 789- 
95.

42. Tadaki S, Sakata Y, Miura Y, et al. Prognostic impacts of metabolic syndrome in patients with chronic heart failure - A multicenter prospective cohort study. Circ J 2016; 80: 677-88.

43. Sakatani T, Shirayama T, Suzaki Y, et al. The association between cholesterol and mortality in heart failure. Comparison between patients with and without coronary artery disease. Int Heart J 2005; 46: 619-29.

44. Tajiri K, Shimizu Y. Branched-chain amino acids in liver diseases. World J Gastroenterol 2013; 19: 7620-9.

45. Samsky MD, Patel CB, DeWald TA, et al. Cardiohepatic inter- actions in heart failure: an overview and clinical implications. J Am Coll Cardiol 2013; 61: 2397-405.

46. Shinagawa H, Inomata $\mathrm{T}$, Koitabashi $\mathrm{T}$, et al. Prognostic significance of increased serum bilirubin levels coincident with cardiac decompensation in chronic heart failure. Circ J 2008; 72: 364-9.

47. Hiraiwa H, Okumura $\mathrm{T}$, Kondo $\mathrm{T}$, et al. Usefulness of the plasma branched-chain amino acid/aromatic amino acid ratio for predicting future cardiac events in patients with heart failure. $\mathrm{J}$ Cardiol 2020; 75: 689-96. 